\title{
Cholangiocarcinoma with intraductal tubular growth pattern versus intraductal papillary growth pattern
}

\author{
Tetsuo Tsukahara ${ }^{1}$, Yoshie Shimoyama ${ }^{2}$, Tomoki Ebata ${ }^{1}$, Yukihiro Yokoyama ${ }^{1}$, \\ Tsuyoshi Igami $^{1}$, Gen Sugawara ${ }^{1}$, Takashi Mizuno ${ }^{1}$, Junpei Yamaguchi ${ }^{1}$, Shigeo Nakamura ${ }^{2}$ \\ and Masato Nagino ${ }^{1}$
}

${ }^{1}$ Division of Surgical Oncology, Department of Surgery, Nagoya University Graduate School of Medicine, Nagoya, Japan and ${ }^{2}$ Department of Pathology and Clinical Laboratories, Nagoya University Graduate School of Medicine, Nagoya, Japan

\begin{abstract}
Intraductal neoplasms of the bile duct are macroscopically characterized by exophytic or polypoid growth patterns and have a favorable prognosis. Although some tumors with a predominantly tubular microscopic pattern have been reported, they have not been well characterized clinicopathologically. The purpose of the present study was to compare the newly recognized cholangiocarcinoma with an intraductal tubular growth pattern and cholangiocarcinoma with an intraductal papillary growth pattern and to investigate the pathological and prognostic significance of the former. This study analyzed 161 patients with tumors with exophytic or polypoid growth patterns from a large series of 733 cholangiocarcinoma cases surgically resected from January 1998 to May 2013. The study patients were divided into two groups: those whose tumors showed a predominantly tubular growth pattern $(n=52)$ and those whose tumors exhibited a predominantly papillary growth pattern $(n=109)$. Tubular growth pattern was associated with combined vascular resection and the absence of macroscopic mucin. Several histological indexes were significantly higher for the tubular growth pattern than the papillary one, including tubular adenocarcinoma, depth of invasion, microscopic lymphatic invasion, venous invasion, perineural invasion, and necrosis. Although the survival curves overlapped $(P=0.693)$, the rate of liver metastasis was significantly higher for the tubular growth pattern than for the papillary one $(P=0.012)$. Genomic DNA analysis focusing on somatic mutations in codons 12 and 13 of KRAS and codon 600 of BRAF revealed only one (4\%) KRAS and no BRAF mutation among the 25 tubular cases examined. In conclusion, the tubular growth pattern exhibited differences in some histologic indexes, in addition to a higher hepatic metastasis rate and a lower KRAS mutation frequency, compared with the papillary growth pattern, but no difference in prognosis was observed. The distinctiveness of this tubular neoplasm should be further examined in the future. Modern Pathology (2016) 29, 293-301; doi:10.1038/modpathol.2015.152; published online 15 January 2016
\end{abstract}

Recent studies have shed new light on cholangiocarcinomas with intraductal proliferative elements, including intraductal papillary neoplasm of the bile duct, ${ }^{1-6}$ which was first documented by Nakanuma et $a l^{7}$ and listed in the 2010 World Health Organization classification. ${ }^{1}$ These tumors are mainly subdivided into two groups, with the intraductal growth type accounting for $6-14 \%$ of

Correspondence: Dr Y Shimoyama, MD, PhD, Department of Pathology and Clinical Laboratories, Nagoya University Graduate School of Medicine, 65 Tsurumai-cho, Showa-ku, Nagoya 466-8550, Japan.

E-mail: simoyama@med.nagoya-u.ac.jp

Received 10 July 2015; revised 17 November 2015; accepted 20

November 2015; published online 15 January 2016 intrahepatic cholangiocarcinomas ${ }^{8-12}$ and the macroscopic papillary growth type accounting for $8-20 \%$ of extrahepatic cholangiocarcinomas. ${ }^{13-15}$ Both types are associated with longer survival than conventional cholangiocarcinoma. ${ }^{8,9,16-18}$

Intraductal papillary neoplasm of the bile duct is characterized by radiographically and macroscopically visible exophytic or polypoid growth pattern with a predominantly papillary growth pattern by histology and varying degrees of tubular and solid components. However, the definition of intraductal papillary neoplasm remains controversial ${ }^{1-6}$ because of the low number of confirmed cases.

A novel type of biliary intraductal neoplasm characterized by a predominantly tubular growth pattern with a focal papillary component has 
recently been documented. ${ }^{19-22}$ Park et $a 1^{19}$ in 2010 reported an unusual intraductal tubulopapillary neoplasm of the bile duct and proposed that their case was not adequately categorized under the current biliary tumor classification. Since their initial report, a few single-case and small-series studies have been published. ${ }^{20-22}$ However, with only 14 total cases reported, the clinical distinctiveness of these tumors remains unproven.

The purpose of the present study was to compare this newly recognized cholangiocarcinoma with an intraductal tubular growth pattern to tumors with an intraductal papillary growth pattern to further our understanding of the clinicopathological features and the prognostic significance of the former.

\section{Materials and methods}

\section{Patients}

This study was approved by the institutional review board. From January 1998 to May 2013, 733 intrahepatic or extrahepatic cholangiocarcinomas were resected surgically at the First Department of Surgery, Nagoya University Hospital. Mucinous cystic neoplasms and papillary lesions of the ampulla of Vater or the gallbladder were excluded. Malignant tumors including combined hepatocellularcholangiocarcinoma, neuroendocrine neoplasms, and sarcomas of any type with intraductal growth were also excluded. We diagnosed 161 patients with prominent exophytic or polypoid growth patterns of the intrahepatic and extrahepatic bile ducts. The macroscopic pattern corresponded to what has been referred to as either the intraductal growth type of intrahepatic cholangiocarcinoma or the papillary growth type of extrahepatic cholangiocarcinoma. The study population included 127 patients with microscopic papillary cholangiocarcinoma examined in our previous study. ${ }^{2}$

Combined vascular resection was defined as reconstruction of the portal vein and/or the hepatic artery. Vascular resection was performed only when the vessel adhered to and could not be freed from the tumor during resection of the hepatoduodenal ligament. Even if invasion was suspected preoperatively, the vessel was not resected when it could be freed from the tumor without difficulty.

\section{Pathologic Examination}

All surgical specimens were sectioned consecutively into 5-mm-thick sections, which were fixed in 10\% formalin and embedded in paraffin. Paraffinembedded blocks were cut into 5 - $\mu$ m-thick sections and stained with hematoxylin and eosin in the usual manner. Formalin-fixed and paraffin-embedded tissue blocks were available in all cases. All histologic slides were retrospectively re-evaluated for the purpose of this study by experienced pathologists.

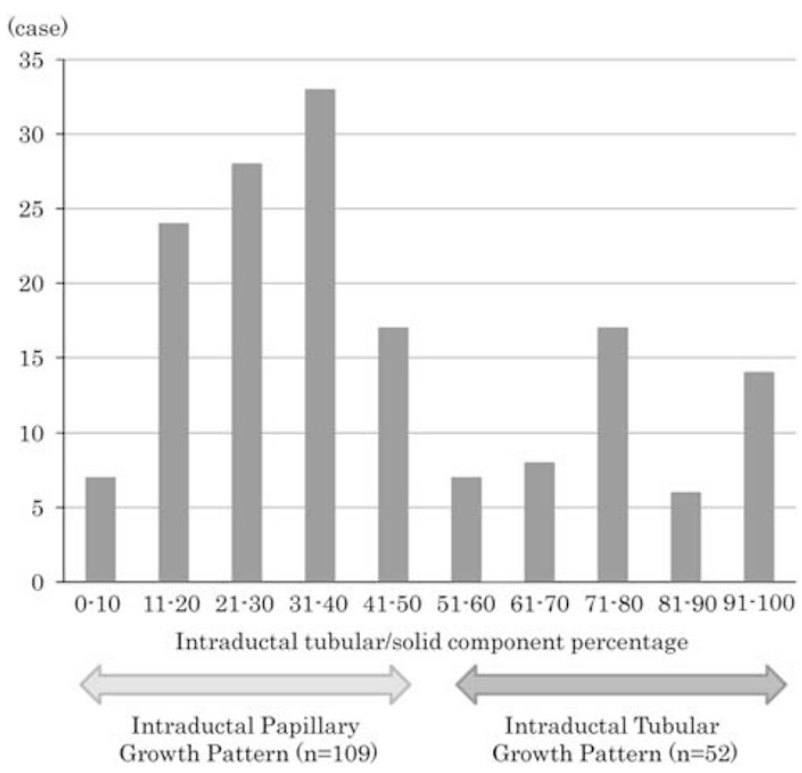

Figure 1 The relationship between intraductal tubular/solid component percentage and cases.

First, tumors with exophytic or polypoid growth patterns were assessed in detail for the intraductal proliferative components associated with papillary, tubular, and solid patterns. Two authors (YS and TT) measured the percentage of microscopic intraductal tubular/solid components in relation to the intraductal tumor volume (intraductal tubular/solid component percentage). According to this percentage, exophytic cholangiocarcinomas were divided into those with a predominantly tubular growth pattern $(n=52)$ and those with a papillary growth pattern ( $n=109$; Figure 1). The cutoff intraductal tubular/ solid component percentage was $50 \%$.

The depth of tumor invasion was measured from the basal lamina of the adjacent normal epithelium to the deepest invasive tumor cells. ${ }^{23}$ The predominant histologic pattern throughout the tumor was used as the representative histologic type of carcinoma. Hepatolithiasis status was also noted. According to our previous report, ${ }^{13}$ superficial spread is defined as noninvasive cancer extension of $>20 \mathrm{~mm}$. With regard to the residual disease status, ductal involvement of noninvasive cancer was considered negative because it had no influence on patient survival. ${ }^{24,25}$ The pathologic findings were described using the TNM Classification of Malignant Tumors, 7th edition, published by the International Union Against Cancer. ${ }^{26}$

\section{Immunohistochemistry}

One to two representative blocks from each case were subjected to immunohistochemical staining for Ki-67, MUC1, MUC2, MUC5AC, human gastric mucin (HGM), MUC6, p53, CDX2, CK7, CK20, and 
CK19. These 11 immunohistochemical stains were performed on tissue sections in all of the cases. The staining patterns in the intraductal proliferative neoplasms of the bile duct were separately documented for tubular/solid and papillary components on selected tissue blocks when both components were present. A binary system, positive or negative, was used to evaluate the staining. The cutoff for positive staining was $>10 \%$ for MUC1, MUC2, MUC5AC, HGM, MUC6, CDX2, CK7, CK20, and CK19, $25 \%$ for p53, and $50 \%$ for Ki-67.

\section{Analyses of Mutations in KRAS and BRAF}

The mutation analysis of the KRAS and BRAF genes was performed by the extraction of genomic DNA from formalin-fixed and paraffin-embedded tumor tissue slides or sections for 25 tumor specimens with a predominantly tubular growth pattern, which were consecutively diagnosed from 2008 until recently. DNA was extracted using a QIAamp DNA FFPE Tissue Kit (Qiagen, Tokyo, Japan) according to the manufacturer's protocol. The scorpion-amplification refractory mutation system method was used for the KRAS gene at codons 12 and 13, and direct sequencing was used for the BRAF gene at codon 600 .

For the scorpion-amplification refractory mutation system assay, KRAS mutations in codons 12 and 13 were detected using a TheraScreen KRAS mutation kit (Qiagen) in accordance with the manufacturer's instructions. This assay detects the G12S, G12C, G12R, G12D, G12V, G12A, and G13D mutations in KRAS. Reactions were conducted in a LightCycler 480 System II (Roche Diagnostics, Tokyo, Japan) and analyzed using LightCycler Adapt software v1.1 (Roche Diagnostics).

Segments of the BRAF gene were amplified by PCR using a GeneAmp PCR System 9700 Thermal Cycler (Life Technologies Japan, Tokyo, Japan). The PCR products were visualized using agarose gel electrophoresis with ethidium bromide staining. PCR DNA fragments were directly sequenced using an ABI 3130xl Genetic Analyzer (Life Technologies Japan) according to the manufacturer's instructions.

\section{Statistics}

Results are expressed as the means \pm s.d. Statistical analyses were performed using the $\chi^{2}$ test, the Fisher's exact test, and the Mann-Whitney $U$ test, where appropriate. Patient survival was determined from the time of surgery to the date of death or the most recent follow-up. Fourteen patients who died of other causes with no evidence of recurrence were treated as censored cases. Postoperative diseasespecific survival was calculated using the KaplanMeier method. Differences in survival curves were compared with the log-rank test. A value of $P<0.05$ was considered to be statistically significant. All calculations were performed using the IBM SPSS
Statistics 22 software package (IBM Japan Inc., Tokyo, Japan).

\section{Results}

\section{Pathologic Findings of Cholangiocarcinoma with Intraductal Tubular Growth Pattern}

Macroscopic findings. Intraductal tubular neoplasms showed prominent exophytic or polypoid growth, forming solid, nodular masses within dilated bile ducts (Figure 2a). The median tumor size was $3.0 \mathrm{~cm}$ in diameter (range, $1.0-8.5 \mathrm{~cm}$ ). The tumors involved the perihilar bile duct in 28 patients, the distal bile duct in 16, and the intrahepatic bile duct in 8 (Table 1).

Microscopic findings. Intraductal tubular cholangiocarcinoma was characterized by intraluminal growth nodules with predominantly or exclusively back-toback tubular glands and a lower degree of papillary components in the bile duct (Figure 2b). Thirty-eight patients $(73 \%)$ with predominantly tubular growth pattern showed a mixture of tubular and papillary components, the latter of which occupied at most $40 \%$ of the intraductal lesion. Solid areas were also observed in seven patients. The neoplastic cells were cuboidal to columnar with enlarged nuclei and eosinophilic or amphophilic cytoplasm (Figure 2c). Cytopathologically, high-grade cellular atypia was observed throughout the tumor, whereas low- or intermediate-grade dysplasia was scarce. Histologically, all cases in the tubular group were invasive tubular adenocarcinomas, which were well (13 patients $(25 \%))$, moderately (26 (50\%)), or poorly differentiated $(10(19 \%))$. The remaining three intraductal tubular cases were diagnosed as mucinous $(n=2)$ and adenosquamous carcinomas $(n=1)$. The mean depth of tumor invasion was $8.1 \mathrm{~mm}$ (range, $0.1-30 \mathrm{~mm}$ ). Extensive mucosal extension of the carcinoma without invasive lesions was observed in 21 patients $(40 \%)$. Three patients exhibited macroscopic luminal mucin secretion, whereas 11 exhibited microscopic mucin production. Necrotic foci were identified in the intraductal component in 28 patients (54\%; Figure 2d). The portal vein and hepatic artery were involved in seven $(13 \%)$, and three patients $(6 \%)$, respectively.

Immunohistochemical findings. The immunohistochemical characteristics are shown in Table 2. The Ki-67 labeling index varied from $2-80 \%$. Of the 52 tubular cases, 34 (65\%) stained positively for MUC1. Staining was positive for MUC2 in $5(10 \%)$ and for MUC5AC in 24 (46\%) patients. All tumors expressed CK7 and/or CK19, supporting ductal differentiation.

Molecular studies. Abnormal expression of p53 was examined using immunohistochemistry. Overexpression of p53 was detected in 15 patients 

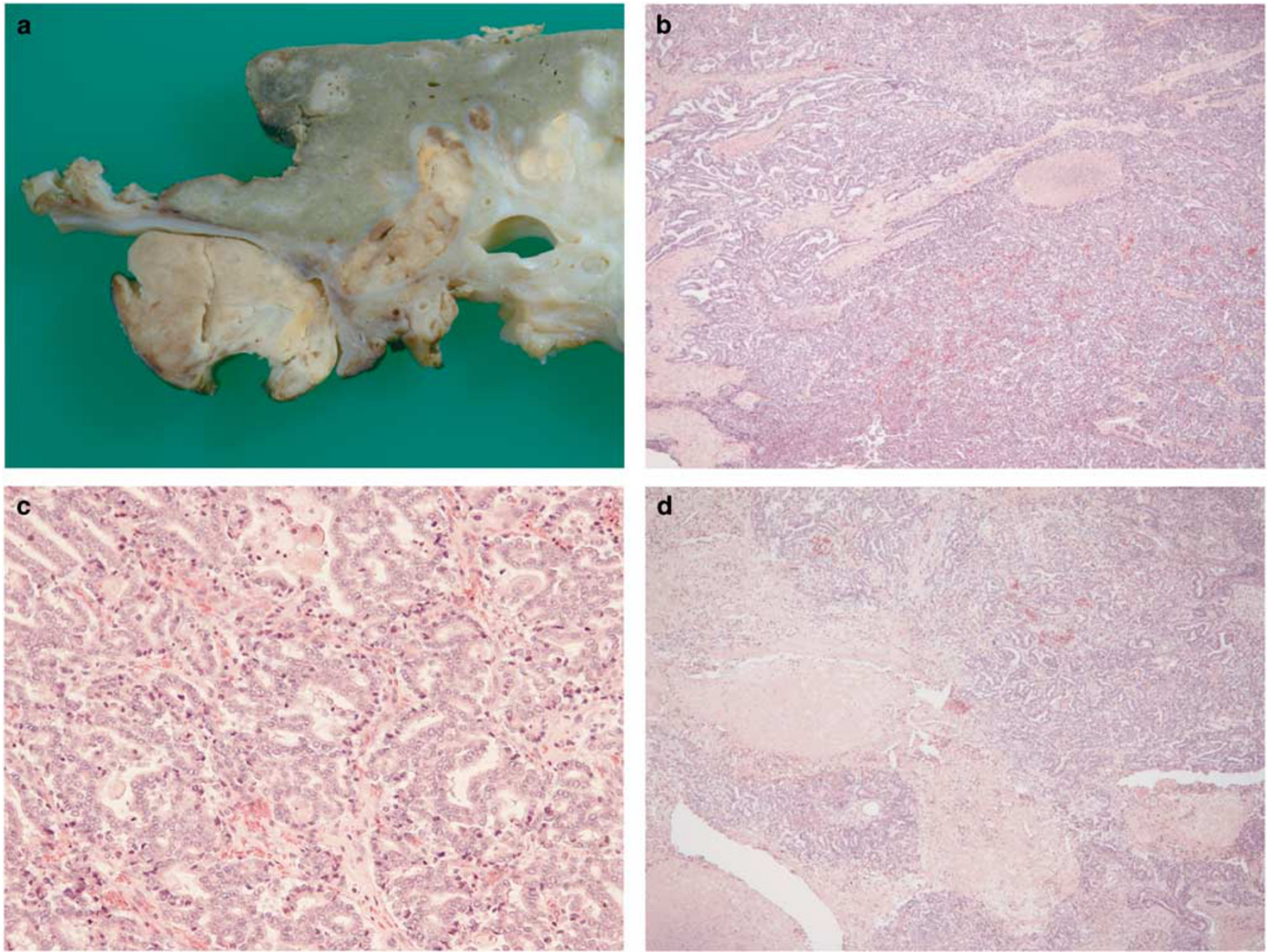

Figure 2 A representative case of cholangiocarcinoma with an intraductal tubular growth pattern. The gross appearance displays a distinct exophytic nodule in the bile duct without mucin secretion (a). The tumor indicates predominantly intraductal tubular proliferation with a focal papillary growth pattern (hematoxylin-eosin (HE), original magnification $\times 40)(\mathbf{b})$. The neoplastic cells are composed of closely back-to-back tubular glands and cuboidal to columnar cells with enlarged nuclei and eosinophilic cytoplasm (HE, original magnification $\times 200)(\mathbf{c})$. Necrotic foci are observed in the intraductal components $(\mathrm{HE}$, original magnification $\times 40)(\mathbf{d})$.

$(29 \%)$ and was more common in extrahepatic cholangiocarcinoma $(15 / 44,34 \%)$ than in intrahepatic cholangiocarcinoma $(0 / 8,0 \% ; P=0.050)$. Somatic mutation in KRAS (G12D) was found in only one patient ( $4 \%$ ), and no BRAF mutations were detected.

\section{Clinicopathological Features of Cholangiocarcinoma with Intraductal Tubular Growth Pattern and Papillary Growth Pattern}

A comparison between 52 tubular cases and 109 papillary cases is shown in Table 1 . In our cohort of 52 tubular cases, 37 cases were male and 15 were female. The age range was between 41 and 93 years with a median age of 67 years. The majority of the patients $(n=31,60 \%)$ presented with symptoms including jaundice $(n=19,37 \%)$, abdominal pain $(n=8,15 \%)$, fever $(n=3,6 \%)$, and weight loss $(n=1$, $2 \%)$; however, 21 patients $(40 \%)$ who had liver dysfunction or were diagnosed incidentally by CT or US performed for other reasons were asymptomatic.

The most common resection was left-sided hepatectomy followed by right-sided hepatectomy in both types of tumors. Both tumor types were usually located in the perihilar bile duct. All of the tubular and papillary cases except for three of the latter had a component of invasive carcinoma. There were no significant differences in age, gender, tumor size, tumor location, T classification, portal vein invasion, hepatic artery invasion, lymph node metastasis, distant metastasis, residual status, or hepatolithiasis between the two groups. Intraductal tubular cholangiocarcinomas were characterized by a higher percentage of combined vascular resection than papillary ones $(25 \%$ vs $10 \%, P=0.013)$, lymphatic invasion ( $56 \%$ vs 39\%, $P=0.039)$, venous invasion (25\% vs $12 \%, P=0.035$ ), perineural invasion (63\% vs $40 \%, P=0.006)$, and necrosis $(54 \%$ vs $29 \%$, $P=0.003)$. The depth of tumor invasion in the tubular type was significantly greater than that in 
Table 1 Clinicopathological features of cholangiocarcinoma with intraductal tubular growth pattern and papillary growth pattern

\begin{tabular}{|c|c|c|c|}
\hline & $\begin{array}{l}\text { Intraductal tubular growth } \\
\text { pattern }(\mathrm{n}=52)\end{array}$ & $\begin{array}{l}\text { Intraductal papillary growth } \\
\text { pattern }(\mathrm{n}=109)\end{array}$ & $\mathrm{P}$-value \\
\hline Age (years) & $65.9 \pm 10.7$ & $67.9 \pm 9.3$ & 0.234 \\
\hline Gender (male/female) & $37 / 15$ & $62 / 47$ & 0.082 \\
\hline Symptoms & & & 0.661 \\
\hline Absent & $21(40 \%)$ & $48(44 \%)$ & \\
\hline Present & $31(60 \%)$ & $61(56 \%)$ & \\
\hline Surgical procedure & & & 0.907 \\
\hline Right-sided hepatectomy & $17(33 \%)$ & $32(29 \%)$ & \\
\hline Left-sided hepatectomy & $23(44 \%)$ & $48(44 \%)$ & \\
\hline Central bisectionectomy & $0(0 \%)$ & $1(1 \%)$ & \\
\hline Partial hepatectomy & $2(4 \%)$ & $2(2 \%)$ & \\
\hline Bile duct resection & $2(4 \%)$ & $6(6 \%)$ & \\
\hline PD without hepatectomy & $8(15 \%)$ & $20(18 \%)$ & \\
\hline Combined vascular resection (present) & $13(25 \%)$ & $11(10 \%)$ & 0.013 \\
\hline Tumor size $(\mathrm{cm})$ & $3.5 \pm 1.8$ & $3.2 \pm 2.0$ & 0.229 \\
\hline Depth of invasion (mm) & $8.1 \pm 7.0$ & $5.1 \pm 5.4$ & 0.003 \\
\hline Location & & & 0.324 \\
\hline Intrahepatic & $8(15 \%)$ & $13(12 \%)$ & \\
\hline Perihilar & $28(54 \%)$ & $72(66 \%)$ & \\
\hline Distal & $16(31 \%)$ & $24(22 \%)$ & \\
\hline Histology & & & $<0.001$ \\
\hline Papillary & $0(0 \%)$ & $56(51 \%)$ & \\
\hline Well & $13(25 \%)$ & $15(14 \%)$ & \\
\hline Moderate & $26(50 \%)$ & $31(28 \%)$ & \\
\hline Poor & $10(19 \%)$ & $1(1 \%)$ & \\
\hline Mucinous & $2(4 \%)$ & $5(5 \%)$ & \\
\hline Adenosquamous & $1(2 \%)$ & $1(1 \%)$ & \\
\hline Lymphatic invasion (present) & $29(56 \%)$ & $42(39 \%)$ & 0.039 \\
\hline Venous invasion (present) & $13(25 \%)$ & $13(12 \%)$ & 0.035 \\
\hline Perineural invasion (present) & $33(63 \%)$ & $44(40 \%)$ & 0.006 \\
\hline T classification & & & 0.397 \\
\hline Tis & $0(0 \%)$ & $3(3 \%)$ & \\
\hline $\mathrm{T} 1$ & $16(31 \%)$ & $35(32 \%)$ & \\
\hline $\mathrm{T} 2$ & $21(40 \%)$ & $44(40 \%)$ & \\
\hline T3 & $10(19 \%)$ & $11(10 \%)$ & \\
\hline $\mathrm{T} 4$ & $5(10 \%)$ & $16(15 \%)$ & \\
\hline Portal vein invasion (present) & $7(13 \%)$ & $9(8 \%)$ & 0.302 \\
\hline Hepatic artery invasion (present) & $3(6 \%)$ & $4(4 \%)$ & 0.405 \\
\hline Lymph node metastasis (present) & $16(31 \%)$ & $23(21 \%)$ & 0.181 \\
\hline Distant metastasis (present) & $5(10 \%)$ & $5(5 \%)$ & 0.186 \\
\hline Residual status & & & 0.492 \\
\hline $\mathrm{R} 0^{\mathrm{a}}$ & $50(96 \%)$ & $103(94 \%)$ & \\
\hline $\mathrm{R} 1 / \mathrm{R} 2$ & $2(4 \%)$ & $6(6 \%)$ & \\
\hline Hepatolithiasis (present) & $0(0 \%)$ & $5(5 \%)$ & 0.138 \\
\hline Superficial spread (present) & $21(40 \%)$ & $51(47 \%)$ & 0.445 \\
\hline Macroscopic mucin (present) & $3(6 \%)$ & $20(18 \%)$ & 0.033 \\
\hline Necrosis (present) & $28(54 \%)$ & $32(29 \%)$ & 0.003 \\
\hline
\end{tabular}

ancluding patients with positive ductal margin with carcinoma in situ.

the papillary type $(P=0.003)$. In the tubular group, macroscopic mucin secretion was significantly less common than in the papillary group $(6 \%$ vs $18 \%$, $P=0.033)$. The rate of superficial spread for the intraductal tubular growth pattern was comparable to the papillary one ( $40 \%$ vs $47 \%, P=0.445$ ).

By immunohistochemical staining, the frequencies of positive MUC1, MUC2, HGM, MUC6, CDX2, CK7, CK20, and CK19 expression in patients with a predominantly tubular growth pattern were similar to those with papillary one (Table 2). The rate of positive MUC5AC in the tubular type was lower than the papillary type, although this difference was not significant $(P=0.067)$. The Ki-67 labeling index and overexpression of p53 showed no significant differences between the two groups $(P=0.582$ and $P=0.853$, respectively).

\section{Survival Analysis of Patients with \\ Cholangiocarcinoma with Intraductal Tubular Growth Pattern and Papillary Growth Pattern}

The median post-surgical follow-up period was 83 months (range, 20-206 months) for all the 
Table 2 Immunohistochemical staining of cholangiocarcinoma with intraductal tubular growth pattern and papillary growth pattern

\begin{tabular}{lccc}
\hline & $\begin{array}{c}\text { Intraductal tubular } \\
\text { growth pattern } \\
(\mathrm{n}=52)\end{array}$ & $\begin{array}{c}\text { Intraductal papillary } \\
\text { growth pattern } \\
(\mathrm{n}=109)\end{array}$ & P-value \\
\hline Ki-67 & $4(8 \%)$ & $8(7 \%)$ & 0.582 \\
MUC1 & $34(65 \%)$ & $64(59 \%)$ & 0.417 \\
MUC2 & $5(10 \%)$ & $15(14 \%)$ & 0.456 \\
MUC5AC & $24(46 \%)$ & $67(61 \%)$ & 0.067 \\
HGM & $24(46 \%)$ & $53(49 \%)$ & 0.769 \\
MUC6 & $15(29 \%)$ & $41(38 \%)$ & 0.275 \\
p53 & $15(29 \%)$ & $33(30 \%)$ & 0.853 \\
CDX2 & $8(15 \%)$ & $16(15 \%)$ & 0.906 \\
CK7 & $48(92 \%)$ & $101(93 \%)$ & 0.582 \\
CK20 & $19(37 \%)$ & $41(38 \%)$ & 0.895 \\
CK19 & $47(90 \%)$ & $101(93 \%)$ & 0.415 \\
\hline
\end{tabular}

161 patients with exophytic growth patterns. Their disease-specific survival rate was $77 \%$ at 3 years and $68 \%$ at 5 years. The median survival was 118 months after resection among these patients. During the follow-up period, 55 patients died of tumor recurrence, 14 patients died of other causes with no evidence of tumor recurrence (pneumonia, $n=4$; cerebral infarction, $n=2$; pancreatic cancer, $n=1$; lung cancer, $n=1$; laryngeal cancer, $n=1$; hepatocellular carcinoma, $n=1$; anaplastic anemia, $n=1$; perforation and bleeding of duodenal ulcer, $n=1$; suicide, $n=1$; and senility, $n=1$ ), 17 patients were alive with recurrent disease, and the remaining 75 patients were alive without disease.

The 52 patients with an intraductal tubular type were followed for a median of 79 months (range, 25-206 months). The 3- and 5-year disease-specific survival rates in the tubular group were $78 \%$ and $70 \%$, respectively. There was no significant difference in disease-specific survival between the two types $(P=0.693$; Figure 3$)$. In our series of tubular lesions, 23 patients are still alive without any evidence of cancer recurrence and 24 patients had postoperative recurrences, including 15 patients with liver metastases, 8 involving other sites, and 1 with unknown sites. The rate of recurrence in tubular cases was similar to that in papillary cases $(46 \%$ vs $44 \%, P=0.801)$. However, the rate of liver metastases in the tubular group (15 of 23 cases with known recurrent sites, $65 \%$ ) was significantly higher than in the papillary group (15 of 45 cases, $33 \% ; P=0.012$ ).

\section{Discussion}

To the best of our knowledge, the current study is the first to compare the clinicopathological features and prognosis of cholangiocarcinoma with an intraductal tubular growth pattern and cholangiocarcinoma with a papillary growth pattern in patients with exophytic or polypoid cholangiocarcinoma. Furthermore, there have been few reports on KRAS and BRAF mutations

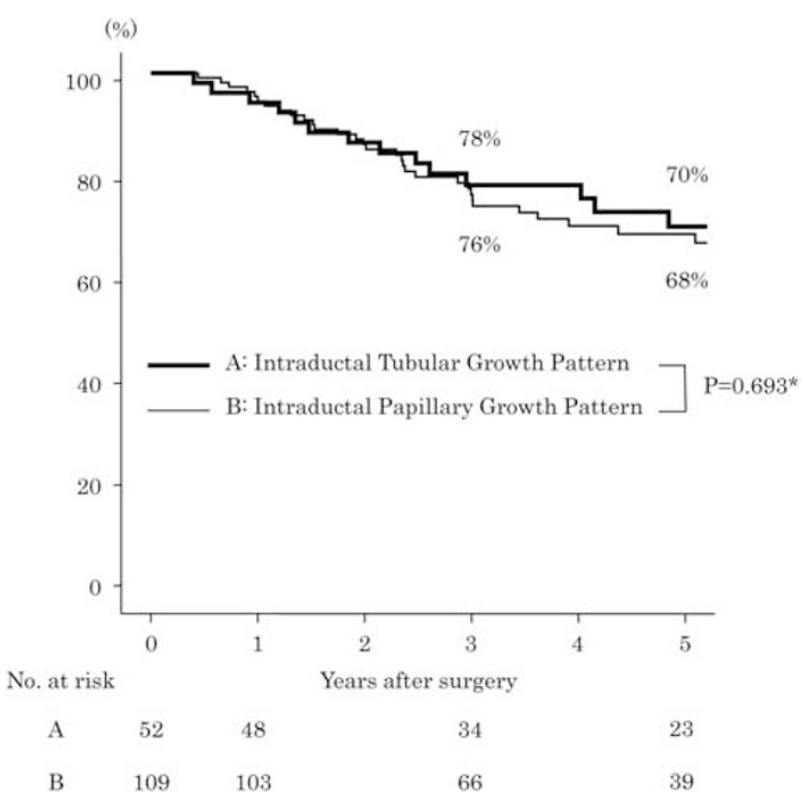

Figure 3 Disease-specific survival of patients with cholangiocarcinoma with an intraductal tubular growth pattern or a papillary growth pattern. *By log-rank test.

in cholangiocarcinoma with intraductal tubular growth.

Among 161 patients with resected cholangiocarcinoma with exophytic or polypoid growth patterns, 52 were categorized as cholangiocarcinomas with a predominantly tubular growth pattern, which were characterized by tubular adenocarcinoma, deeper tumor invasion, microscopic lymphatic invasion, venous invasion, perineural invasion, the absence of macroscopic mucin, and necrosis (Table 1). No differences were found in the expression of immunohistochemical stains between the intraductal tubular type and the papillary type (Table 2). The 5-year survival rate for patients in the tubular group was comparable to that in the papillary group $(70 \% \mathrm{vs}$ $68 \%, P=0.693$; Figure 3 ). However, the rate of liver metastases in tubular cases was significantly higher than in papillary cases $(P=0.012)$. These metastases may be related to microscopic venous invasion, the rate of which was significantly higher in the tubular group than in the papillary group $(P=0.035$; Table 1). These findings suggest that intraductal tubular cholangiocarcinomas may be somewhat different from papillary ones. However, our sample size is too small to draw any definite conclusions.

Increasing interest has been focused on intraductal papillary neoplasms in pancreatobiliary diseases. In the bile duct, according to the present World Health Organization classification, ${ }^{1}$ intraductal neoplasms are classified into biliary intraepithelial neoplasia and intraductal papillary neoplasm of the bile duct, which have been proposed to represent two individual precursor lesions preceding invasive cholangiocarcinoma. Biliary intraepithelial neoplasias are flat or low-papillary lesions with varying degrees 
of cellular atypia, and they are recognized microscopically. ${ }^{27,28}$ They are known to progress to conventional cholangiocarcinoma with invasion into the ductal wall and to express MUC2 partially and MUC5AC widely. ${ }^{4,5,29}$ Conversely, intraductal papillary neoplasms of the bile duct are composed of macroscopically visible, intraductal papillary projections, which have grossly visible mucin secretion in one-third of cases, and which display four types of epithelium: pancreatobiliary, intestinal, gastric, and oncocytic. Intraductal papillary neoplasms express MUC2 and MUC5AC widely. ${ }^{3-5}$ In the present study, cholangiocarcinomas with an intraductal papillary growth pattern were predominantly intraductal papillary neoplasms of the bile duct. However, the clinicopathological features of cholangiocarcinoma with an intraductal tubular growth pattern appear to be substantially different from biliary intraepithelial neoplasia and intraductal papillary neoplasm of the bile duct. Thus, this tumor does not pertain to any categories in the current biliary tumor classification.

In 2010, Park et $a I^{19}$ reported an unusual intraductal tubulopapillary neoplasm of the bile duct with lung metastasis. Zen et $a l^{22}$ also described two cases of intraductal tubulopapillary neoplasms of the bile duct in association with peribiliary cysts, which had wild-type genotypes for KRAS and BRAF. Katabi et $a l^{20}$ subsequently reported 10 cases of intraductal neoplasms showing a predominantly tubular growth pattern, defined as having $>50 \%$ tubular/solid architecture, with a papillary component. We also attempted to stratify exophytic cholangiocarcinomas into those with a predominantly tubular growth pattern $(n=52)$ or a predominantly papillary one $(n=109)$, depending on the intraductal tubular/solid component percentage, similar to Katabi et al..$^{20}$ Our data were generally in accordance with the findings previously documented in their case series with some differences. The similar points are briefly summarized as follows: (1) the appearance of a macroscopically solid nodular tumor obstructing dilated ducts; (2) intraductal tubular growth with a focal papillary component; (3) uniform high-grade atypia throughout the neoplasm; (4) occasional foci of necrosis; and (5) ductal differentiation as indicated by CK7 and/or CK19 expression. The differences are as follows: (1) no visible mucin secretion (macroscopic mucin production was observed in three patients in our study); (2) absence of MUC2 and MUC5AC (positive MUC2 and MUC5AC expression was found in 5 and 24 patients, respectively, in our study); and (3) no overexpression of p53 (overexpression detected in 15 patients of our series). These discrepancies might be related to our inclusion of heterogeneous tumors into the tubular group. It has also been speculated that a novel type of biliary intraductal neoplasm with a predominantly tubular architecture may be analogous to intraductal tubulopapillary neoplasm of the pancreas. ${ }^{30}$ This issue should be clarified in the future.
In our study, a few genetic analyses were performed. Immunolabeling for p53 protein was found in 15 of 52 tubular cases (29\%). Moreover, genomic DNA was analyzed for somatic mutations in codons 12 and 13 of KRAS and codon 600 of $B R A F$ in 25 of the 52 patients with the intraductal tubular type. Both KRAS and BRAF are members of the RAS-dependent mitogen-activated protein kinase pathway, which mediates cellular responses to growth signals. ${ }^{31} K R A S$ belongs to the RAS family that encode guanosine-5'-triphosphate-binding proteins as an effector of ligand-bound EGFR. BRAF encodes a serine-threonine protein kinase that is a downstream effector of activated KRAS. Somatic mutations in KRAS were found in only one patient (4\%), and no mutations in BRAF were observed. In previous studies, biliary intraepithelial neoplasia and conventional cholangiocarcinomas have shown mutation rates of $13-59 \%$ in $K R A S^{32-36}$ and $0-22 \%$ in $B R A F .^{33,34,36,37}$ Intraductal papillary neoplasm of the bile duct has exhibited mutation rates of $18-46 \%$ in $K R A S,{ }^{38,39}$ but there are no reports on $B R A F$ mutations. KRAS mutations have been occasionally found in biliary intraepithelial neoplasia, intraductal papillary neoplasm of the bile duct, and conventional cholangiocarcinomas, but they have been rarely observed in cholangiocarcinomas with an intraductal tubular growth pattern. The frequency of KRAS mutations in intraductal tubular cholangiocarcinomas appears to be much lower than in other cholangiocarcinomas. These data may highlight some differences in the molecular oncogenesis between these neoplasms, and they suggest potential targets for molecular diagnosis and therapy of cholangiocarcinoma. Further molecular analyses will be required.

The present study has some limitations. First, it was retrospective and involved a rather limited number of patients. Second, it remains controversial whether intraductal tubular growth pattern and papillary one constitute a continuous spectrum or whether they represent heterogeneous subgroups of exophytic growth patterns with different tumorigenesis. Further studies with a larger number of cases are needed to explore these issues.

In conclusion, cholangiocarcinoma with a predominantly tubular growth pattern is an uncommon cancer of the bile duct with an intraductal exophytic growth pattern, representing $\sim 7 \%$ of all resectable cases. Intraductal tubular cholangiocarcinoma shows differences in some histologic indexes, in addition to a higher hepatic metastasis rate and a lower KRAS mutation frequency, but it has a similar prognosis compared with papillary one.

\section{Disclosure/conflict of interest}

The authors declare no conflict of interest. 


\section{References}

1 Nakanuma Y, Curado M, Franceschi S et al. Intrahepatic cholangiocarcinomaBosman FT, Carneiro F, Hruban RH et al. WHO Classification of Tumours of the Digestive System. 4th edn Lyon, France: IARC. 2010; 217-224.

2 Onoe S, Shimoyama Y, Ebata $\mathrm{T}$ et al. Prognostic delineation of papillary cholangiocarcinoma based on the invasive proportion: a single-institution study with 184 patients. Surgery 2014;155:280-291.

3 Zen Y, Pedica F, Patcha VR et al. Mucinous cystic neoplasms of the liver: a clinicopathological study and comparison with intraductal papillary neoplasms of the bile duct. Mod Pathol 2011;24: 1079-1089.

4 Zen Y, Fujii T, Itatsu K et al. Biliary papillary tumors share pathological features with intraductal papillary mucinous neoplasm of the pancreas. Hepatology 2006;44:1333-1343.

5 Zen Y, Sasaki M, Fujii T et al. Different expression patterns of mucin core proteins and cytokeratins during intrahepatic cholangiocarcinogenesis from biliary intraepithelial neoplasia and intraductal papillary neoplasm of the bile duct-an immunohistochemical study of 110 cases of hepatolithiasis. J Hepatol 2006;44: 350-358.

6 Ohtsuka M, Kimura F, Shimizu H et al. Similarities and differences between intraductal papillary tumors of the bile duct with and without macroscopically visible mucin secretion. Am J Surg Pathol 2011;35: 512-521.

7 Chen TC, Nakanuma Y, Zen Y et al. Intraductal papillary neoplasia of the liver associated with hepatolithiasis. Hepatology 2001;34:651-658.

8 Tajima Y, Kuroki T, Fukuda K et al. An intraductal papillary component is associated with prolonged survival after hepatic resection for intrahepatic cholangiocarcinoma. Br J Surg 2004;91:99-104.

9 Morimoto Y, Tanaka Y, Ito T et al. Long-term survival and prognostic factors in the surgical treatment for intrahepatic cholangiocarcinoma. J Hepatobiliary Pancreat Surg 2003;10:432-440.

10 Yamasaki S. Intrahepatic cholangiocarcinoma: macroscopic type and stage classification. J Hepatobiliary Pancreat Surg 2003;10:288-291.

11 Shirai K, Ebata T, Oda K et al. Perineural invasion is a prognostic factor in intrahepatic cholangiocarcinoma. World J Surg 2008;32:2395-2402.

12 Yamamoto M, Takasaki K, Yoshikawa $\mathrm{T}$ et al. Does gross appearance indicate prognosis in intrahepatic cholangiocarcinoma? J Surg Oncol 1998;69:162-167.

13 Sakamoto E, Nimura Y, Hayakawa N et al. The pattern of infiltration at the proximal border of hilar bile duct carcinoma: a histologic analysis of 62 resected cases. Ann Surg 1998;227:405-411.

14 Todoroki T, Kawamoto T, Koike $\mathrm{N}$ et al. Radical resection of hilar bile duct carcinoma and predictors of survival. Br J Surg 2000;87:306-313.

15 Bhuiya MR, Nimura Y, Kamiya J et al. Clinicopathologic factors influencing survival of patients with bile duct carcinoma: multivariate statistical analysis. World J Surg 1993;17:653-657.

16 Albores-Saavedra J, Murakata L, Krueger JE et al. Noninvasive and minimally invasive papillary carcinomas of the extrahepatic bile ducts. Cancer 2000;89: $508-515$.
17 Yeh TS, Tseng JH, Chen TC et al. Characterization of intrahepatic cholangiocarcinoma of the intraductal growth-type and its precursor lesions. Hepatology 2005;42:657-664.

18 Nakanuma Y, Sato Y, Harada K et al. Pathological classification of intrahepatic cholangiocarcinoma based on a new concept. World J Hepatol 2010;2: 419-427.

19 Park HJ, Jang KT, Heo JS et al. A potential case of intraductal tubulopapillary neoplasms of the bile duct. Pathol Int 2010;60:630-635.

20 Katabi N, Torres J, Klimstra DS. Intraductal tubular neoplasms of the bile ducts. Am J Surg Pathol 2012;36: 1647-1655.

21 Sato Y, Osaka H, Harada K et al. Intraductal tubular neoplasm of the common bile duct. Pathol Int 2010;60: 516-519.

22 Zen Y, Amarapurkar AD, Portmann BC. Intraductal tubulopapillary neoplasm of the bile duct: potential origin from peribiliary cysts. Hum Pathol 2012;43: $440-445$.

23 Hong SM, Cho H, Moskaluk CA et al. Measurement of the invasion depth of extrahepatic bile duct carcinoma: An alternative method overcoming the current T classification problems of the AJCC staging system. Am J Surg Pathol 2007;31:199-206.

24 Igami T, Nagino M, Oda K et al. Clinicopathologic study of cholangiocarcinoma with superficial spread. Ann Surg 2009;249:296-302.

25 Wakai T, Shirai Y, Moroda T et al. Impact of ductal resection margin status on long-term survival in patients undergoing resection for extrahepatic cholangiocarcinoma. Cancer 2005;103:1210-1216.

26 Sobin LH, Gospodarowicz MK, Wittekind C. International Union Against Cancer. TNM Classification of Malignant Tumours. 7th (edn). Hoboken, NJ, USA: Wiley-Blackwell. 2010.

27 Zen Y, Adsay NV, Bardadin K et al. Biliary intraepithelial neoplasia: an international interobserver agreement study and proposal for diagnostic criteria. Mod Pathol 2007;20:701-709.

28 Zen Y, Aishima S, Ajioka Y et al. Proposal of histological criteria for intraepithelial atypical/proliferative biliary epithelial lesions of the bile duct in hepatolithiasis with respect to cholangiocarcinoma: preliminary report based on interobserver agreement. Pathol Int 2005;55:180-188.

29 Higashi M, Yamada N, Yokoyama S et al. Pathobiological implications of MUC16/CA125 expression in intrahepatic cholangiocarcinoma-mass forming type. Pathobiology 2012;79:101-106.

30 Yamaguchi H, Shimizu M, Ban S et al. Intraductal tubulopapillary neoplasms of the pancreas distinct from pancreatic intraepithelial neoplasia and intraductal papillary mucinous neoplasms. Am J Surg Pathol 2009;33:1164-1172.

31 Peyssonnaux C, Eychene A. The Raf/MEK/ERK pathway: new concepts of activation. Biol Cell 2001;93: 53-62.

32 Ohashi K, Tstsumi M, Nakajima Y et al. Ki-ras point mutations and proliferation activity in biliary tract carcinomas. Br J Cancer 1996;74:930-935.

33 Tannapfel A, Sommerer F, Benicke M et al. Mutations of the BRAF gene in cholangiocarcinoma but not in hepatocellular carcinoma. Gut 2003;52:706-712.

34 Andersen JB, Spee B, Blechacz BR et al. Genomic and genetic characterization of cholangiocarcinoma 
identifies therapeutic targets for tyrosine kinase inhibitors. Gastroenterology 2012;142:1021-1031.

35 Hsu M, Sasaki M, Igarashi S et al. KRAS and GNAS mutations and p53 overexpression in biliary intraepithelial neoplasia and intrahepatic cholangiocarcinomas. Cancer 2013;119:1669-1674.

36 Voss JS, Holtegaard LM, Kerr SE et al. Molecular profiling of cholangiocarcinoma shows potential for targeted therapy treatment decisions. Hum Pathol 2013;44:1216-1222.
37 Goldenberg D, Rosenbaum E, Argani P et al. The V599E BRAF mutation is uncommon in biliary tract cancers. Mod Pathol 2004;17:1386-1391.

38 Matthaei H, Wu J, Dal Molin M et al. GNAS codon 201 mutations are uncommon in intraductal papillary neoplasms of the bile duct. HPB (Oxford) 2012;14: 677-683.

39 Sasaki M, Matsubara T, Nitta T et al. GNAS and KRAS mutations are common in intraductal papillary neoplasms of the bile duct. PLoS One 2013;8:e81706. 